\title{
Investigation of Robotic Material Loading Strategies using an Earthmoving Simulator
}

\author{
Eric Halbach Aarne Halme Ville Kyrki \\ Department of Electrical Engineering and Automation, Aalto University, Finland \\ eric.halbach@gmail.com, \{firstname.lastname\} @aalto.fi
}

\begin{abstract}
A kinematic earthmoving simulation environment was used to investigate job planning strategies which could increase the performance of automated material loading with a robotic compact skid-steered wheel loader. One new problem studied was the subdivision of a larger rectangular workspace using the smaller rectangular Scoop Area (SA). Two methods for selecting scooping approach vectors were also compared: a Zero Contour (ZC) method which assesses all possible perpendicular approaches along the bottom of the slope, and the proposed alternative High Point (HP) method which scoops towards the highest point in the current workspace from a fixed point. Three jobs were simulated to determine which scooping method and SA dimensions resulted in the highest excavation rate in a truck loading scenario. Assuming the same scoop filling effectiveness, the HP method was found to offer a higher rate than the $\mathrm{ZC}$ method due to its more limited driving envelope. The maximum HP rates were achieved with SA dimensions which were narrower and longer than with the ZC method, while the optimal SA dimensions were also found to be dependent on the job parameters. When a higher amount of material to excavate per area was present, smaller SAs resulted in higher rates.
\end{abstract}

Keywords: automation, robotics, earthmoving, excavation, wheel loader, simulation, job planning

\section{Introduction}

Using robotic earthmoving machinery at mining and construction sites offers the possibility of both increasing safety and lowering costs. By separating human operators from the worksite, exposure to potential hazards such as collisions, rockfalls, dust and fumes is reduced, while commuting times can also be cut by controlling machinery from the safety and comfort of an office, which could be located far from the site.

This leads to the question of how such robotic machines would be controlled. By automating parts of the load-haul-dump work cycle and limiting direct teleoperation, efficiency can be increased by allowing one human to monitor and/or control several machines. Some commercial systems such as Sandvik's AutoMine and Caterpillar's Command for Underground already make this a reality by automating the hauling and dumping segments of the work cycle, though the loading or excavation phase usually needs to be controlled by a skilled human operator, possibly remotely by teleoperation.

Automating the excavation or loading phase could further increase efficiency by enabling fully automated work cycles. This is made difficult, however, by the unpredictable reaction forces encountered in ground material, which can contain fragments of unknown sizes and behave differently depending on factors such as compaction and moisture. Despite this challenge, solutions have been proposed for autonomous bucket filling, with some demonstrations being performed using full-sized machinery (Lever and Wang, 1995; Sarata et al., 2008; Almqvist, 2009; Dobson et al., 2015).

Fully automated work cycles could also make systems applicable in situations where direct teleoperation is not possible due to a long telecommunication time delay, such as in some space applications (e.g. 4-21 minutes to Mars one-way). Earthmoving capabilities on other planetary bodies would be useful for establishing a permanent human presence, for jobs such as settlement construction and harvesting regolith for resource extraction (Mackenzie et al.; Petrov, 2004). Given the additional hazards of radiation exposure and risk of depressurization which humans would face operating in these environments, supervising fully automated robots from Earth may be the ideal case for such jobs. Even if humans are located on site, however, full automation would be desirable for reducing human workload and freeing the crew for other important tasks.

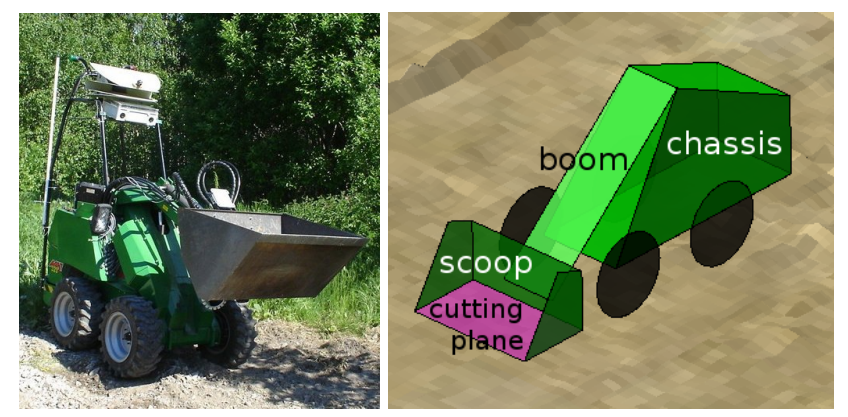

Figure 1. Compact skid-steered Avant 320 (left) and virtual model (right).

Assuming that scooping can be controlled automatically, a higher-level planning requirement for automated 
earthmoving is deciding where to dig within a designated workspace such that progress is made towards the goal state. It may also be desirable to optimize some criterion, such as maximizing the excavation rate or minimizing energy use. This paper presents simulations for investigating this problem in the case of automated material loading by a robotic compact skid-steered wheel loader, modeled after an Avant 320 which was available to the authors for testing (see Figure 1).

The next section begins by presenting related work in this research area, followed by a description in Section 3 of the simulation environment used. Section 4 presents the strategy developed for subdividing a large rectangular workspace using the smaller rectangular Scoop Area (SA) in a truck loading scenario. Two methods for generating scooping approaches are then described in Section 5: the Zero Contour (ZC) method which selects a perpendicular approach along the bottom edge of the slope, and the proposed alternative High Point (HP) method which scoops towards the highest point in the workspace from a fixed point. Section 6 presents simulation results of jobs which were repeated using various SA dimensions, and both scooping methods, to observe the effect on the excavation rate. Different job parameters were used to also investigate the effect of less surrounding slope collapse and a higher slope. The conclusion and areas for future work are then discussed in Section 7.

\section{Related Work}

The work in this paper is partly based on the multiresolution planning for robotic earthmoving developed by Singh and Cannon, which first subdivides a larger workspace with a coarse planner, then select digging locations with a refined planner (Singh and Cannon, 1998). Their planning for a wheel loader assumes the presence of an independently positioned dump truck which is to be filled, with the scooping actions limited to a region near the truck. Scooping actions are made perpendicular to the zero contour, or bottom edge of the slope, to achieve even loading of the bucket. All scooping locations are assessed before selecting one based on maximizing contour convexity into the scoop (to ease loading), minimizing side load (for an even fill) and minimizing the distance to the truck (Singh and Cannon, 1998).

Sarata et al. demonstrated automated scooping and truck loading cycles with a full-size wheel loader (Sarata et al., 2008). With their method, scooping locations are also located at the zero contour, with the scooping action oriented so as to minimize the predicted side moment on the bucket, to reduce wear. For the next action, the point a certain distance to the right or left of the previous one is chosen which is feasible and minimizes the hauling distance (Sarata et al., 2005).

Magnusson and Almqvist extend the work of Singh and Cannon for wheel loaders by using a more complex bucket model, and by evaluating convexity and side load along the entire bucket fill trajectory (Magnusson and Almqvist, 2011). Magnusson et al. also developed a coarse-to-fine planner and show how it ensures the long-term availability of good scooping locations as a large pile is excavated (Magnusson et al., 2015).

The ZC method implemented in this paper is a simplified 2D version of that proposed by Singh and Cannon (Singh and Cannon, 1998). It serves as an example of a method that selects from a large number of feasible actions along the contour, and is compared with the simple proposed HP method.

The second main planning investigation in this paper has so far not been found in the literature, though it has been alluded to (Singh and Cannon, 1998). I.e. in the case of dump truck locations which are dependent on excavating a slope evenly, which sub-region dimensions should be used to optimize some desired criterion (such as the excavation rate)?

\section{Earthmoving Simulator}

The robotic earthmoving strategies were investigated using a simulation environment developed using Matlab, based on previous work by the authors (Halbach and Halme, 2013; Halbach, 2007). It is similar to that used by Sarata and Magnusson et al. (Sarata, 2001; Magnusson et al., 2015), and allows ground material to be removed and deposited while maintaining a maximum angle of repose and conserving the total volume of material (thereby assuming a constant material density).

The simulator is purely kinematic and does not model forces, an approach taken for simplicity and because it was not intended for developing control of scooping actions, but rather for developing high-level planning strategies such as where to dig and where to dump material as a worksite changes over time. It therefore offers a compromise between the physics-based approach used in other simulators (Bonchis et al., 2011; Schmidt et al., 2010; PlaCastells et al., 2009), and simulators developed primarily for visualization of construction processes which do not necessarily conserve the amount of material (Kamat and Martinez, 2005; Lipman and Reed, 2000).

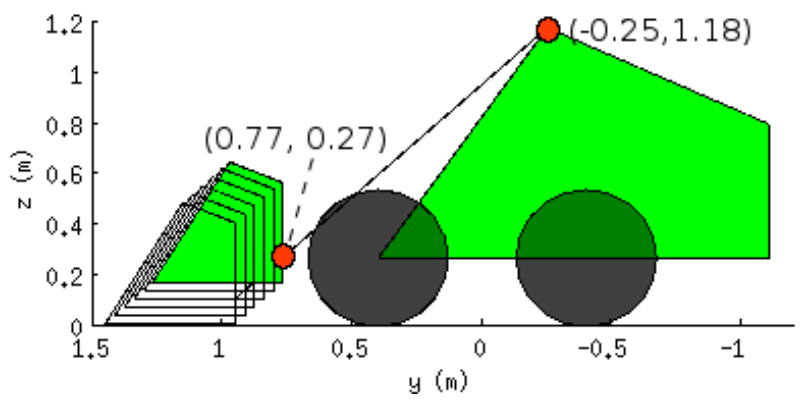

Figure 2. Avant model kinematics and range of scooping configurations resulting from extension of prismatic boom joint.

In this environment, a worksite is modeled as a surface with a $0.1 \mathrm{~m}$ grid resolution. The Avant 320 model 
(see Figure 1) has wheels spaced $0.79 \mathrm{~m}$ width-wise and axles spaced $0.80 \mathrm{~m}$ apart, with the wheels, rendered as 2D disks, representing the centres of the tires. The vehicle's location and heading angle are defined in the XYplane, while the current pose is determined from the average height of the four surface points at the $2 \mathrm{D}$ wheel locations, and the average slope between these points.

Machines in the simulator are assumed to possess accurate positioning and autonomous driving capabilities. Driving occurs by turning on the spot at a rate of $30 \%$ and following straight paths at $0.5 \mathrm{~m} / \mathrm{s}$. One timestep in the simulator is $1 / 3 \mathrm{~s}$.

The scoop has dimensions $0.89 \mathrm{~m}$ wide by $0.5 \mathrm{~m}$ long, and a volume capacity set at $0.15 \mathrm{~m}^{3}$. Three joints are available for scoop positioning: rotary, between the chassis and boom; telescopic, for extending the boom; and rotary, between the boom and bucket. Figure 2 shows kinematic details of the joint locations, with the vehicle (reference point in the middle of the wheels at ground level) at $y=0$, and the boom and bucket in their home positions.

Scoop-ground interaction works by checking for intersections between the bottom "cutting plane" of the scoop and the ground surface at each time step. Figure 3 (left) shows how the cutting plane is discretized with the red circular points. If any of the blue square ground points are above the corresponding point in the cutting plane (as in Figure 3, middle), the ground point is lowered and the column volume above added to the scoop load.
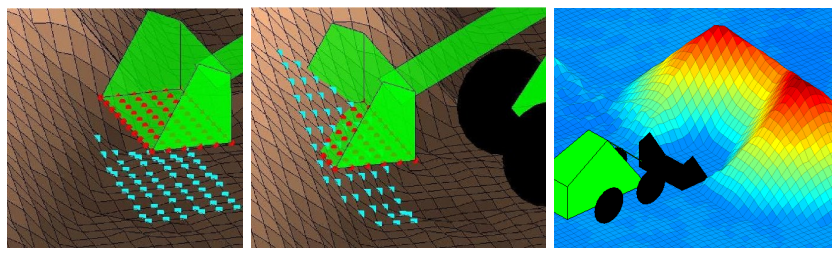

Figure 3. Checking for intersection of discretized cutting plane points (red circles) with corresponding ground points (blue squares) during scooping action (left, centre); result of simulated slope collapse (right).

Scooping actions are performed with the cutting plane level (both rotary joints in home position), with a boom extension ranging from $0 \mathrm{~m}$ to $0.24 \mathrm{~m}$, corresponding to the bottom of the scoop positioned $0.17 \mathrm{~m}$ to $0.01 \mathrm{~m}$ above the ground (see Figure 2). The value to use for the next action is determined automatically at the end of each current action by comparing the scoop height with the desired ground level. If the scoop ended up too high or low, the boom setting is extended or retracted accordingly by $0.01 \mathrm{~m}$ for the next scooping action. Although the next action is usually at a different location, this strategy generally helps to maintain the designated scooping area at ground level. This strategy is necessary due to the kinematic nature of the simulator, which allows ground heights to be lowered by any intersection with the cutting plane. In a dynamic environment this strategy may not be necessary since the bucket could collide with and/or scrape along the ground, and a constant preset scooping configuration might be possible.

During a scooping action, material is added to the scoop load until the time step at which the current volume increment would cause the scoop capacity to be surpassed. At this point, a certain minimum fill ratio is assumed, with the remaining scoop capacity filled randomly and any leftover material deposited back on the ground. With a minimum of 0.8 , for example, an average of 0.9 results over many actions. This strategy was developed so that it would be possible to specify the average performance of the scooping controller, which is assumed to exist, while allowing for some random effects due to tool-ground interaction.

When the bucket is raised after a scooping action, slope collapse is simulated by scanning in the $\mathrm{X}$ and $\mathrm{Y}$ directions for slope sections which are above the maximum specified repose angle. These are then adjusted (conserving volume), and neighbouring sections checked, until stability is reached. Figure 3 (right) shows the result of this simulated soil behaviour after several scooping actions into a pile. It is assumed that after any changes to the surface occur, the ground model would be updated by onboard laser scanners or by other surveyor robots.

\section{Workspace Subdivision with Scoop Area}

If excavating material from a large area with a wheel loader, the best coarse planning strategy may depend on where the material is to be deposited. If the dumping location is a stationary bin, then the loader would be free to select any location along the entire dig face - the scenario studied by Magnusson et al. (Magnusson et al., 2015). If dump trucks are being loaded, then a smaller digging region near the truck should generally be used to reduce the amount of driving between digging actions. Singh and Cannon studied the case of an independently positioned truck (Singh and Cannon, 1998), however here it is assumed that the main requirement is to excavate the slope face evenly, with the dump trucks positioned as needed to accomplish this goal.

Another assumption is that the workspace is rectangular, thus the method followed to excavate the slope evenly is to scan the workspace in a raster pattern from front to back with the smaller rectangular Scoop Area (SA), shown in Figure 4 mid-way through Job 1a. This job consists of excavating an $11 \times 2.4 \mathrm{~m}$ section out of a $0.87 \mathrm{~m}$ high plateau with a $30^{\circ}$ slope. When a location is found which has ground heights a certain threshold (here $0.15 \mathrm{~m}$ ) above ground level, the loader works there until the SA is cleared, and the SA then scans for the next location, with the machines repositioning there.

The graphical objects rendered in Figure 4 are interactive planning tools developed previously by the authors (Halbach and Halme, 2013). The large rectangular surface is used to specify and visualize the full workspace, while the triangular prism marks the Approach Side (AS). 


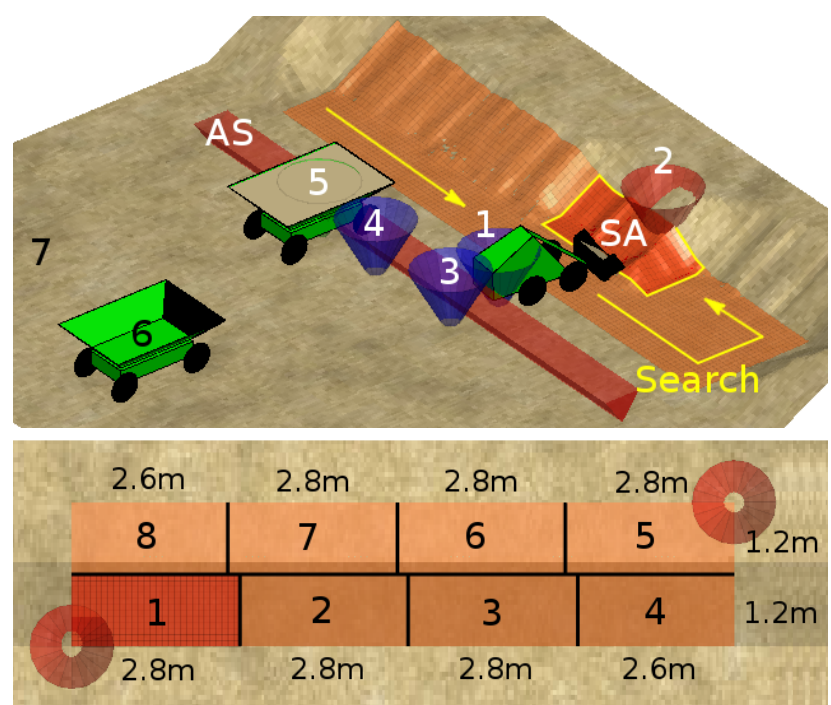

Figure 4. Scoop Area (SA) scans workspace for next working location along raster pattern from front to back; SA target dimensions (here $3 \times 1 \mathrm{~m}$ ) adjusted to divide into workspace (11 x $2.4 \mathrm{~m})$ evenly.

A scooping action begins at the Stage point (cone 1) and is directed towards the Scooping Destination (cone 2), with the loader reversing to point 1 after the load is extracted.

Cones 3 and 4 represent driving waypoints for load transfer to the dump truck (at cone 5), which has a load capacity of $1 \mathrm{~m}^{3}$ and is also skid-steered. A $2^{\text {nd }}$ dump truck waits at point 6 , and when one truck is filled, it drives to point 7 where the load is deleted, and continues to point 6 , while the other truck drives to point 5 . Points 6 and 7 would be the end of a hauling road along which the loads are transported, though this is not included here. The points are positioned relative to the current SA location. It should be noted that these planning strategies are specific to skid-steered machines which can turn on the spot.

The SA scans for the next working location with steps of one width and length. Its intended "target" dimensions are sometimes altered by an algorithm which attempts to divide the full workspace by the SA dimensions evenly, to avoid SA locations which only contain a fractional amount of work. The bottom of Figure 4 shows how the workspace is divided using target dimensions of $3 \times 1 \mathrm{~m}$.

\section{High Point and Zero Contour Meth- ods}

This section describes the two methods for generating scooping approach vectors which are compared. The ZC method is based on the work of Singh and Cannon (Singh and Cannon, 1998), and selects a perpendicular approach along the bottom edge of a slope after evaluating convexity and the distance to point 3 (see Figure 4). A zero contour is first constructed by searching the workspace for points a certain threshold (here $0.15 \mathrm{~m}$ ) above ground level, then following the contour until either the edge of the workspace is reached or the contour is closed. Figure 5 shows an example of contours constructed around an irregular pile shape.

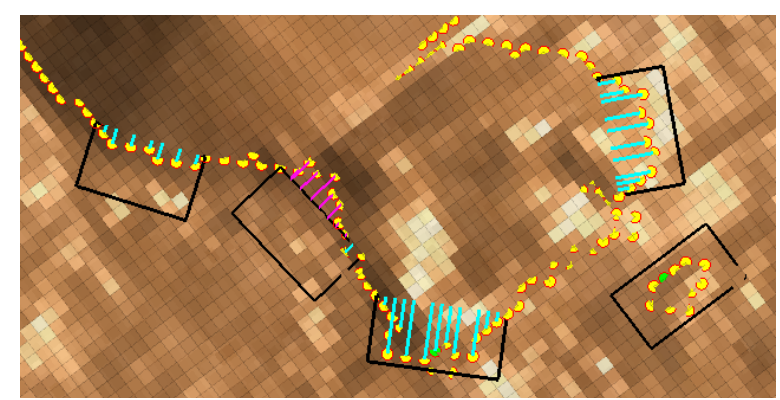

Figure 5. Convexity evaluation at possible scooping locations along zero contour; small separate contour at right assigned one possible location.

All possible scooping locations are then assessed by tracing along the contours with a line segment as wide as the scoop, with each end of the segment touching the contour. The convexity at each location is determined in $2 \mathrm{D}$ by adding the perpendicular line segments of points in between which protrude past the line, and subtracting those beyond the line (blue and purple lines in Figure 5). A possible scooping location is selected if its convexity is over $10 \%$ greater than the best value found so far (to attempt increasing filling effectiveness), or if it is within $10 \%$ of the best value and closer to point 3 (to reduce driving). Approaches which have a backwards-facing heading are not considered (to avoid excessive maneuvering), nor are those with non-traversable paths. If no acceptable scooping locations can be found, the HP method is used as a backup (described next).

In Figure 6 the $\mathrm{ZC}$ method is being used to excavate an SA location in Job 1a, with the yellow points showing the contour. At left is a new SA location, with a scooping approach selected at the corner which maximizes convexity. At right an unloading action is shown, and the different shape of the contour is also evident after 9 actions.
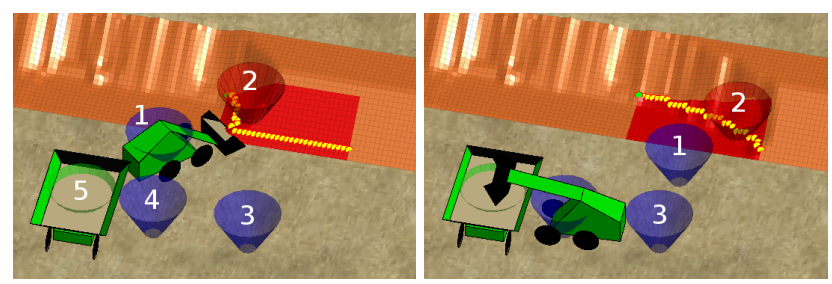

Figure 6. Excavation of SA with ZC method, with new Stage point (1) and Scooping Destination (2) for each scooping action; (left) first action at new SA, (right) after 9 actions with unloading at truck illustrated.

The HP method is a simple alternative which was proposed in previous work by the authors (Halbach and Halme, 2013), whereby the Stage point remains stationary and scooping actions are directed towards the highest point in the SA (see Figure 4). This results in a fan-shaped 
pattern as the highest point shifts due to slope collapse, illustrated at left in Figure 7. In this example from Job 1a, 18 actions were needed to level the SA location. This coverage pattern can be compared with that using the $\mathrm{ZC}$ method at right, which consisted of 19 actions to clear the SA. The ZC driving paths appear to require more turning and driving, since they approach the SA more from the left side.

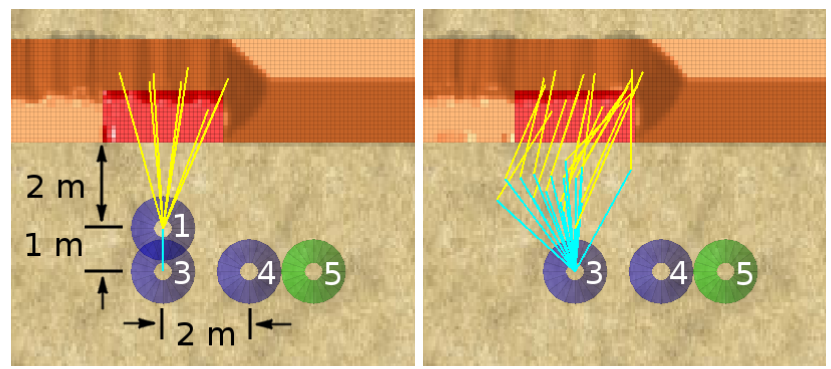

Figure 7. Coverage pattern for leveling $2.8 \times 1.2 \mathrm{~m} \mathrm{SA}$ location with HP method (left) using 18 actions, and ZC method (right) using 19 actions.

The HP method was not originally intended to be an improvement over others that have been proposed, but was meant to be a simple way of generating commands in order to test excavation jobs in the simulator. Its real-world effectiveness, which would need to be tested, may be hindered by the fact that it does not consider contour convexity or side loading, and can result in non-perpendicular approaches into the slope. If it could work well enough in practice, however, it may offer the benefit of reduced total driving, which is investigated next.

\section{Simulation Results and Discussion}

To find the SA dimensions which result in the maximum excavation rate, Job 1a was repeated with various SA widths and lengths, using both scooping methods. The minimum scoop filling ratio was kept constant at 0.8 (average fill of 0.9 ), thus a main assumption is that both the $\mathrm{HP}$ and $\mathrm{ZC}$ methods perform with the same scooping effectiveness.

A constant plateau height was chosen for this job in an attempt to reduce the factors which could affect the excavation rate, so that the SA dimensions would be the main variables during each simulation. With this constantheight plateau, each row excavated should have the same amount of material collapsing in from uphill, though the amount collapsing from the sides would initially increase as the front slope is excavated.

Two more versions of Job 1 were simulated to observe the effect of less surrounding slope collapse and a higher plateau (see Figure 8). Job 1b (at left) is a stand-alone plateau which fits in the same $11 \times 2.4 \mathrm{~m}$ workspace, and has the same height and slope angle. Job 1c (at right) is similar to Job 1a, with the same workspace dimensions, however with the plateau height doubled to $1.73 \mathrm{~m}$.

The excavation rate results for these jobs are plotted in
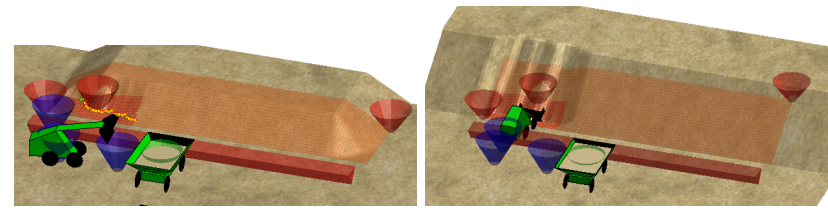

Figure 8. Job $1 \mathrm{~b}$ (left), $0.87 \mathrm{~m}$-high stand-alone plateau or pile, and Job 1c (right), $1.73 \mathrm{~m}$-high plateau, both with $30^{\circ}$ slopes.

Figure 9. Included in these plots are the ranges of SA dimensions which were chosen manually to show the rise and fall of the rate, with the width on the X-axis and different lines plotted for each length value. Each point is the average rate recorded after repeating the job 10 times, which was assumed to be sufficient given the randomness introduced in the scoop filling. The error bars represent one standard deviation.
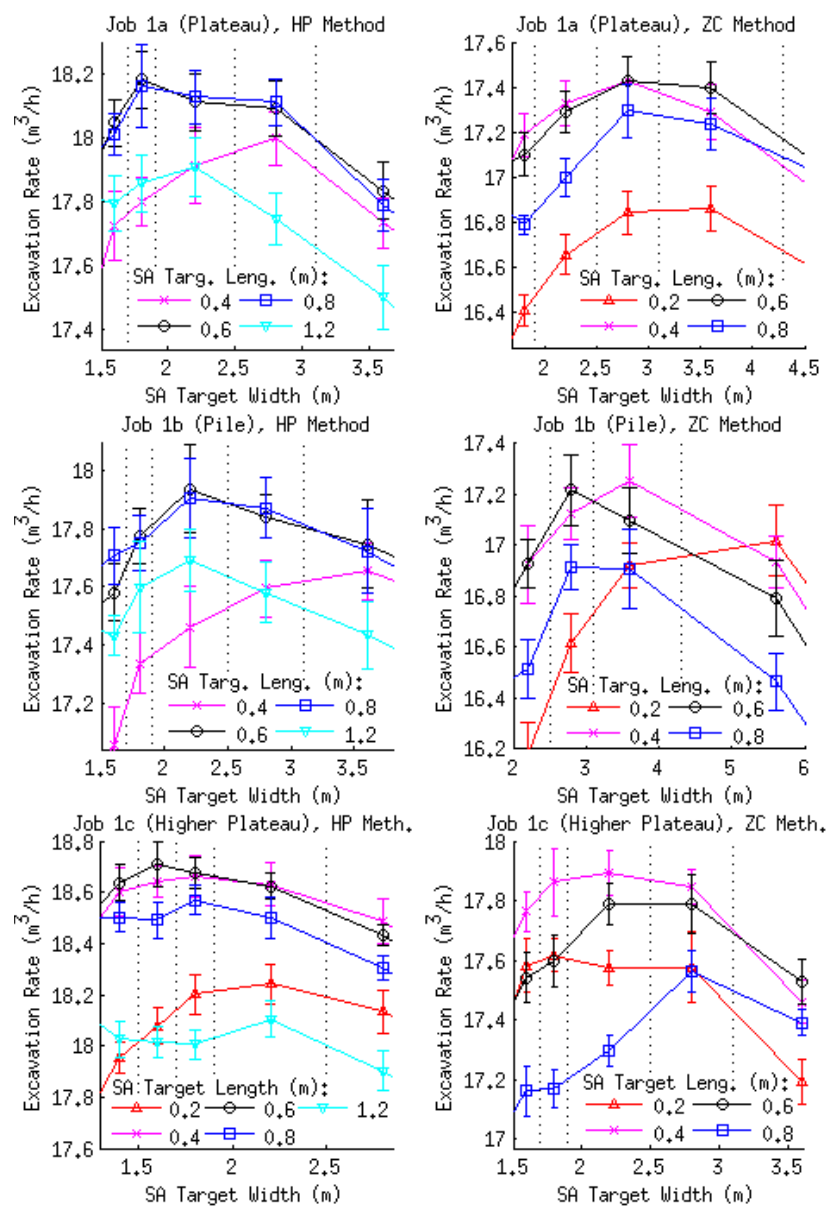

Figure 9. Excavation rate for Jobs 1a-c with varying SA target dimensions using HP and ZC method; 0.8 minimum scoop load ratio; 10 trials per data point.

In these plots, each data point represents a range of target SA dimensions which map to that value due to the algorithm which attempts to divide the workspace evenly. For the width dimension, the ranges are represented by the dotted lines. Target SA widths of 2.0-2.4 m, for example, map to a width of $2.2 \mathrm{~m}$ (5 SA locations along $11 \mathrm{~m}$ width). Similarly, a line for the target length of $1.0 \mathrm{~m}$ is 
Table 1. Maximum Excavation Rate (Rate 1) and Volume per Combined Drive Time (Rate 2) for Jobs 1a-c

\begin{tabular}{|c|c|c|c|c|c|c|}
\hline \multirow[b]{2}{*}{ Job } & \multicolumn{3}{|c|}{ HP Method } & \multicolumn{3}{|c|}{ ZC Method } \\
\hline & $\begin{array}{l}\text { Max. } \\
\text { Rate 1 } \\
\left(\mathrm{m}^{3} / \mathrm{h}\right)\end{array}$ & $\begin{array}{l}\text { Target } \\
\text { Width } \\
\text { (m) }\end{array}$ & $\begin{array}{l}\text { Target } \\
\text { Length } \\
\text { (m) }\end{array}$ & $\begin{array}{l}\text { Max. } \\
\text { Rate } 1 \\
\left(\mathrm{~m}^{3} / \mathrm{h}\right)\end{array}$ & $\begin{array}{l}\text { Target } \\
\text { Width } \\
\text { (m) }\end{array}$ & $\begin{array}{l}\text { Target } \\
\text { Length } \\
\text { (m) }\end{array}$ \\
\hline $1 \mathrm{a}$ & 18.182 & 1.8 & 0.6 & 17.433 & 2.8 & 0.4 \\
\hline $1 b$ & 17.938 & 2.2 & 0.6 & 17.253 & 3.6 & 0.4 \\
\hline $1 \mathrm{c}$ & 18.710 & 1.6 & 0.6 & 17.891 & 2.2 & 0.4 \\
\hline & $\begin{array}{l}\text { Max. } \\
\text { Rate } 2 \\
\left(\mathrm{~m}^{3} / \mathrm{h}\right)\end{array}$ & $\begin{array}{l}\text { Target } \\
\text { Width } \\
\text { (m) }\end{array}$ & $\begin{array}{l}\text { Target } \\
\text { Length } \\
\text { (m) }\end{array}$ & $\begin{array}{l}\text { Max. } \\
\text { Rate } 2 \\
\left(\mathrm{~m}^{3} / \mathrm{h}\right)\end{array}$ & $\begin{array}{l}\text { Target } \\
\text { Width } \\
\text { (m) }\end{array}$ & $\begin{array}{l}\text { Target } \\
\text { Length } \\
\text { (m) }\end{array}$ \\
\hline $1 \mathrm{a}$ & 15.519 & 2.8 & 0.8 & 15.003 & 3.6 & 0.6 \\
\hline $1 b$ & 15.148 & 2.8 & 0.8 & 14.722 & 5.6 & 0.6 \\
\hline $1 \mathrm{c}$ & 15.999 & 2.2 & 0.6 & 15.458 & 2.8 & 0.6 \\
\hline
\end{tabular}

not plotted since this is mapped to $1.2 \mathrm{~m}$. The maximum rates and corresponding SA dimensions for each method and version of Job 1 are summarized in Table 1.

The table also includes results with the volume per combined drive time measure (plots not shown), which is the volume excavated divided by the total driving and turning time of the loader and two dump trucks. This is included to consider the case where excavating with minimal driving would be more important than excavating quickly, e.g. if energy is limited such as in a planetary construction scenario.

One observation is that in each case, the HP method achieves a higher maximum rate than the $\mathrm{ZC}$ method, likely due to the HP method's more limited coverage pattern with less turning and driving. This, again, assumes the same bucket filling effectiveness for both methods.

Another observation is that with the HP method, the maximum rates are achieved with SAs which are narrower and longer than with the $\mathrm{ZC}$ method. One reason for this could be that after unloading at the truck, with the HP method the loader always turns $90^{\circ}$ at point 3 to reach point 1 (see Figure 7), therefore narrower SAs may be preferred to reduce further turning. The $\mathrm{ZC}$ method may prefer shorter SAs because they cannot contain contours with much curvature, and more curvature could result in more maneuvering to approach from the side. Short SAs would then need to be wider to contain enough material so that the SA does not reposition too frequently, which increases driving. Since with the ZC method the loader turns at point 3 by varying amounts towards the moving Stage point, far ends of wider SAs can perhaps be reached sooner than with the HP method.

It can also be observed when comparing the different job versions that when there is more material to excavate per area, such as with the higher plateau of Job 1c compared with Job 1a, or with more surrounding slope collapse in Job 1a compared with Job 1b, higher rates result. These are also usually achieved with smaller SAs, likely because with more material to excavate per area, smaller SAs become beneficial since they can be covered with less driving. Smaller SAs have the disadvantage of more repositioning of the machines between SA locations, however this is evidently outweighed by the advantage of less driving within the SAs.

Finally, the optimal SA dimensions with the volume per combined drive time measure are larger than with the standard excavation rate. This could be expected, since although larger SAs require more driving within them, they also require less repositioning between SAs. Repositioning would impose a bigger penalty with this measure since it involves all three machines driving simultaneously.

\section{Conclusions and Future Work}

The simulation results presented in this paper showed that the HP method resulted in higher excavation rates than the $\mathrm{ZC}$ method for various slope excavation jobs. One area for future work would be to check if a real loader could indeed fill its bucket as effectively with the HP method, despite the possible drawback of occasional non-perpendicular approach vectors which could result in asymmetrical loading. Future work would also include implementing the system with robotic machines and demonstrating the necessary site modeling, autonomous driving and scooping control capabilities.

The ZC method tended to reach its maximum rates with SA dimensions which were wider and shorter than with the HP method. It was also found that when more material was present per area, due to a higher plateau or more surrounding slope collapse, smaller SAs resulted in higher excavation rates. For reducing total machine driving, larger SAs were beneficial.

In general applications, piles and slopes could have irregular shapes and heights, thus as another area for future work, an algorithm could be developed which first analyzes the properties of the slope to excavate, then estimates optimal SA dimensions. During the job, the dimensions could be adjusted automatically based on the observed slope properties, or also in a speculative way to see if a higher rate can be achieved.

\section{Acknowledgment}

The authors would like to acknowledge the Academy of Finland for funding the Centre of Excellence in Generic Intelligent Machines (GIM) (2008-2013), of which this research was a part, and also the Graduate School in Electronics, Telecommunications and Automation (GETA) for supporting this work (2011-2013).

\section{References}

Håkan Almqvist. Automatic bucket fill. Master's thesis, Linköping University, 2009.

Adrian Bonchis, Nicholas Hillier, Julian Ryde, Elliot Duff, and Cédric Pradalier. Experiments in Autonomous Earth Moving. 
In 18th International Federation of Automatic Control (IFAC) World Congress, Milan, Italy, 2011.

Andrew A. Dobson, Joshua A. Marshall, and Johan Larsson. Admittance Control for Robotic Loading: Underground Field Trials with an LHD. In Field and Service Robotics, Toronto, Canada, 2015.

Eric Halbach. Development of a Simulator for Modeling Robotic Earth-Moving Tasks. Master's thesis, Helsinki University of Technology, 2007.

Eric Halbach and Aarne Halme. Job planning and supervisory control for automated earthmoving using 3D graphical tools. Automation in Construction, 32:145-160, 2013.

Vineet R. Kamat and Julio C. Martinez. Large-Scale Dynamic Terrain in Three-Dimensional Construction Process Visualizations. Journal of Computing in Civil Engineering, 19(2): 160-171, 2005.

Paul J. A. Lever and Fei-Yue Wang. Intelligent Excavator Control System for Lunar Mining System. Journal of Aerospace Engineering, 8(1):16-24, 1995.

Robert Lipman and Kent Reed. Using VRML in Construction Industry Applications. In 5th Symposium on Virtual Reality Modeling Language (VRML), Monterey, U.S.A., 2000.

Bruce Mackenzie, Bart Leahy, Georgi Petrov, and Gary Fisher. The Mars Homestead: a Mars Base Constructed from Local Materials. In Space 2006, San Jose, U.S.A.

Martin Magnusson and Håkan Almqvist. Consistent Pile-Shape Quantification for Autonomous Wheel Loaders. In IEEE/RSJ International Conference on Intelligent Robots and Systems (IROS), San Francisco, U.S.A., 2011.

Martin Magnusson, Tomasz Kucner, and Achim J. Lilienthal. Quantitative Evaluation of Coarse-to-Fine Loading Strategies for Material Rehandling. In IEEE International Conference on Automation Science and Engineering (CASE), Gothenburg, Sweden, 2015.

Georgi I. Petrov. A Permanent Settlement on Mars: The First Cut in the Land of a New Frontier. Master's thesis, Massachusetts Institute of Technology, 2004.

Marta Pla-Castells, Ignacio García-Fernández, Miguel A. Gamón, and Rafael J. Martínez-Durá. Interactive earthmoving simulation in real-time. In Congreso Español de Informática Gráfica (CEIG) (Spanish Congress of Computer Graphics), San Sebastián, Spain, 2009.

Shigeru Sarata. Model-based Task Planning for Loading Operation in Mining. In IEEE/RSJ International Conference on Intelligent Robots and Systems (IROS), Maui, U.S.A., 2001.

Shigeru Sarata, Yossewee Weeramhaeng, and Takashi Tsubouchi. Planning of scooping position and approach path for loading operation by wheel loader. In 22nd International Symposium on Automation and Robotics in Construction (IS$A R C)$, Ferrara, Italy, 2005.
Shigeru Sarata, Noriho Koyachi, and Kazuhiro Sugawara. Field Test of Autonomous Loading Operations by Wheel Loader. In IEEE/RSJ International Conference on Intelligent Robots and Systems (IROS), Nice, France, 2008.

Daniel Schmidt, Martin Proetzsch, and Karsten Berns. Simulation and Control of an Autonomous Bucket Excavator for Landscaping Tasks. In IEEE International Conference on Robotics and Automation (ICRA), Anchorage, U.S.A., 2010.

Sanjiv Singh and Howard Cannon. Multi-Resolution Planning for Earthmoving. In IEEE International Conference on Robotics and Automation (ICRA), Leuven, Belgium, 1998. 